\title{
A Comparison Between the Effects of Manual Therapy Plus Sexual Advice With Manual Therapy Alone in Patients With Low Back Pain: A Study Protocol
}

Musa Sani Danazumi ( $\square$ musadanazumisani@gmail.com )

Department of Physiotherapy, College of Health Sciences, Faculty of Allied Health Sciences, Bayero University, Kano, Nigeria, https://orcid.org/0000-00023084-5552

Abdulsalam Mohammed Yakasai

Department of Physiotherapy, College of Health Sciences, University of KwaZulu-Natal, Durban, South Africa

Shehu Usman Ibrahim

Department of Physiotherapy, College of Health Sciences, Faculty of Allied Health Sciences, Bayero University, Kano, Nigeria

Usman Tijjani Shehu

Department of Public Health, School of Health Sciences, University of Salford Manchester, United Kingdom

Mubarak Falke Abubakar

Department of Physiotherapy, Federal Medical Centre, Nguru, Yobe State, Nigeria

Usman Usman Zakari

Department of Physiotherapy, Federal Medical Centre, Birnin Kudu, Jigawa State, Nigeria

Method Article

Keywords: Manual therapy, Sexual advice, Low back pain, Radiculopathy

Posted Date: July 2nd, 2020

DOI: https://doi.org/10.21203/rs.3.rs-37636/v1

License: (c) (1) This work is licensed under a Creative Commons Attribution 4.0 International License. Read Full License 


\section{Abstract}

Objective: The aim of this study is to compare the effects of manual therapy plus sexual advice (MT+SA) with manual therapy alone (MTA) in the management of individuals with low back pain (LBP).

Methods: A total of 208 individuals diagnosed with LBP secondary to lumbar disk herniation with radiculopathy (LDHR) will be randomly (computer-generated block randomization) assigned into 2 treatment groups; 104 participants each in (MT+SA) and (MTA) groups. Each participant will be assessed at baseline, 4 , 8 and 12 weeks of intervention and then at 6, 9, and 12 months of follow-up. Outcomes to be assessed will include both self-report (pain, disability, sciatica bothersomeness and sciatica frequency), and objective outcomes (gait speed and dynamic isometric abdominal strength). Repeated measures ANOVA with intervention (MT+SA and MTA) as the between-subject variable and time (weeks 4, 8 and 12, months 6, 9 and 12) as the within-subject variable will be used to analyze treatment effects.

Discussion: This study will determine if the addition of sexual advice to manual therapy will yield better outcomes than when manual therapy alone is administered to individuals with LBP secondary to LDHR.

Trial Registration: Pan African Clinical Trial Registry: PACTR202002785212512.

\section{Hightlights}

- Sexual intercourse can lead to and/or aggravate low back pain (LBP).

- Sexual intercourse is a significant measure of disability and health-related quality of life.

- There is paucity of studies that recognize sexual advice as part of rehabilitation program of LBP.

- If the integration of sexual advice in LBP yields better outcomes, then it may be included.

\section{Introduction}

Individuals with low back pain (LBP) are usually taught how to perform activities of daily living, but infrequently are they counseled on how to perform sex. ${ }^{1}$ Sex can be very essential just like other activities, but sometimes difficult to talk about. ${ }^{2}$ Talking about sex has become a thing of shy and so many of our clients suffer its detrimental effects at the expense of our shyness. At times, it is absurd and ridiculous to talk about sex, but believe it as you may also argue that sex and life are part and parcel of each other and sex forms one of the central aspects of every relationship. ${ }^{3}$

Even though inappropriate sexual contact can cause LBP, the actual statistic is yet to be examined because the problem is not widely reported. ${ }^{1}$ However, studies 4,5 indicate that $34-84 \%$ of individuals with LBP pain experienced a decline in the rate of sexual activity and sex was accountable for increasing the pain in $64 \%$ of individuals with LBP. ${ }^{5}$ In addition, the most commonly experienced problems during sex by individuals with LBP were finding suitable sexual positions and problems with hip movements. ${ }^{4}$

Studies ${ }^{6,7}$ have indicated that sexual behavior or ejaculation decreases pain sensitivity by stimulating the release of endogenous opioids (EOs) peptides, potentially to avoid tight sexual arousal from becoming unpleasant and raise its reward value. Sexual intercourse is highly linked to analgesia in humans ${ }^{8}$ and rats ${ }^{9,10}$ and is also rewarding. ${ }^{11}$ This may indicate the possibility of developing or exacerbating LBP obliviously during sexual intercourse as the partners may not likely recognize any pain due to opioids' analgesia.

Sexual intercourse has been identified as a significant indicator of people's lives and one of the inherent measures of health and disability and can be as much a part of the human experience as eating and drinking. ${ }^{2}$ If sex is an activity in which people with LBP are interested, there is no reason to limit them. Unfortunately, in spite of the high prevalence of decreased sexual activity in individuals with LBP, there are quite a few studies ${ }^{12,131}$ on how to enjoy peaceful sexual intercourse which is necessary for proper back hygiene and overall wellbeing of the individuals.

In addition, there is to date no any study that included sexual advice as part of its treatment protocol for individuals with LBP which suggest that novel studies are urgently warranted in this field. For this reason, this study will for the first time be conducted to examine the effects of manual therapy as an adjunct to sexual advice than manual therapy alone in the management of individuals with LBP. It was hypothesized that manual therapy in combination with sexual advice will yield better outcomes than manual therapy alone in the management of individuals with LBP.

\section{Materials And Methods}

\section{Research Design and Ethical Issues}

This study will be designed as a single-blind parallel randomized clinical trial (RCT); the research participants will be blinded to randomization and group allocation but the investigators will not be blinded because of the nature of the study. However, outcome assessors (research assistants) will be blinded. Ethical concerns in this study will be treated in accordance with Declaration of the Helsinki. Ethical approval to conduct this study was obtained from the Health Research Ethics Committee of Federal Medical Centre, Nguru, Yobe state, Nigeria - FMC/N/CL.SERV/355/VOLvi/191. Participants will be assured of their rights to withdraw from the study at any point in time and their full anonymity will be maintained. The trial was registered with the Pan African Clinical Trial Registry — PACTR202002785212512. 


\section{Sample Size and Sampling Technique}

The sample size was calculated using the $\mathrm{G}^{*}$ Power version 3.1 software. ${ }^{14}$ The effect size used for calculating the sample size was obtained from the previous study ${ }^{15}$ using disability outcome (modified Oswestry low back pain questionnaire-MOLBPQ) after 6-weeks of intervention. The formula that was used to compute the effect size was; $\eta 2=\mathrm{SS}_{\mathrm{B}} / \mathrm{SS}_{\mathrm{T}}$. The effect size was calculated using the following inputs $-\mathrm{SS}_{\mathrm{B}}=1781.062$, and $\mathrm{SS}_{\mathrm{T}}=4683.035$. The calculation yielded a total sample size of 174 participants (87 per group) using the following inputs; tail (1), probability level (a-0.05), power ( $\mathrm{p}-0.8$ ), effect size (d-0.38), allocation ratio (N2/N1 - 1), for t-test of difference between two independent means (two groups). However, according to a previous study ${ }^{16}$ $20 \%$ attrition rate is needed to be added in a clinical trial of equal allocation to groups. For this reason, the sample size was adjusted to 208 ( $174 \mathrm{X} 20 / 100$ ) participants (104 per group) to cover up for attrition rate. The sampling method that will be used to obtain the required number of the participants in this study will be a consecutive sampling method. Consecutive sampling method was chosen because the participants may not all be present at once.

\section{Eligibility Criteria}

Participants diagnosed (MRI confirmed and/or physical examination) as having low back pain due to Lumbar Disc Herniation with Radiculopathy (LDHR) of any duration will be included in the study. Participants must be sexually active (not having impotence) and having active sexual partners (this is to ensure that participants can have sex anytime they needed it). Participants will be assessed for erectile dysfunctions (ED) using the 5 -Items version of the International Index of Erectile Function (IIEF-5). The IIEF-5 is an instrument used to determine the presence and extent of ED. ${ }^{17}$ This Questionnaire consists of only five questions and each IIEF- 5 item is scored on a five-point ordinal scale. A response of 1 indicates least sexual function, whereas a response of 5 indicates highest sexual function. The highest possible cumulative score for the IIEF- 5 is 25 , while the least score is 1 . A score above 21 will be considered as a normal erectile function and a score at or below this value will be considered as ED. ${ }^{17}$ The exclusion criteria will include; 1 ) participants diagnosed as having other back pathologies not LDHR, 2) participants diagnosed as having LDHR but with commobidities and/or red flags (vertebral collapse, osteoporosis, previous spinal surgery, pregnancy, cognitive impairment, cancer, sudden weight loss, recent infection, bladder and/or bowel dysfunction), 3) participants diagnosed as having LDHR but not sexually active (IIEF-5 score of $\leq 21$ ) and not having healthy sexual partners.

\section{Data Collection Procedure}

\section{Participants Recruitment}

Participants will be recruited from the orthopedic and medical outpatient departments of XXXXX, to the physiotherapy department of the same hospital where the study will be conducted. Participants will be screened for eligibility by orthopedic physiotherapists. Participants who meet the eligibility criteria and willing to participate will be recruited for the study. The participants will be given an informed consent form to sign after which their demographic and clinical parameters will be obtained.

\section{Outcomes Assessment}

Both self-report and objective outcomes will be assessed in this study because a previous study ${ }^{18}$ indicated that discrepancies exist between these outcomes in individuals with LDHR. These outcomes will be assessed at baseline, 4, 8 and 12 weeks of intervention. Participants will be discharged home after 12-weeks of intervention and be placed on lumbar stabilization exercises ${ }^{19}$ as home regimen. Outcomes will also be assessed at 6,9 and 12 months of follow-up.

\section{Participants' Self-Report Measures}

1. Visual Analogue Scale (VAS): This parameter will be used to measure the intensity of the pain in the back and leg experienced by the research subjects. Assessment will be based on a horizontal $100 \mathrm{~mm}$ scale varying from $0 \mathrm{~mm}$, 'no pain in the back', to $100 \mathrm{~mm}$, 'the worst pain ever'. These parameters will be included because a lot of patients with LDHR used to have both back and leg pain in varying intensities, which can change after surgery or conservative treatment. ${ }^{20,21}$ VAS has been shown to have high inter-observer reliability coefficient $(r=0.88) .{ }^{22}$

2. Roland Morris Disability Questionnaire (RMDQ) for Sciatica: This illness-specific 23-item functional assessment questionnaire will be used to measure functional limitation. Scores range from 0 to 23, reflecting a simple un-weighted sum of items to be endorsed by the respondents. Patients with high scores at baseline will be categorized as having severe disabilities. The Roland Questionnaire for Sciatica has a documented high level of internal consistency reliability (Cronbach's alpha of 0.87 ), test-retest reliability (ICC of 0.9 ) construct validity, and responsiveness to change. ${ }^{23}$

3. Sciatica Scales: Sciatica bothersomeness will be assessed using the Sciatica Bothersomeness Index (SBI) and sciatica frequency will be assessed using the Sciatica Frequency Index (SFI). These will be done by asking the participants to complete the entire questionnaires at the beginning of the study (baseline assessment). Outcomes will also be assessed at 6 and 12 weeks after which participants will be discharged home and placed on therapeutic exercises as home regimen. These questionnaires have high level of internal consistency reliability (Cronbach's alpha of 0.74 for SBI and 0.70 for SFI) and test-retest reliability (ICC of 0.86 for SBI and 0.90 for SBI). ${ }^{24}$

\section{Participants' Objective Measures}

1. Gait Speed: Timed Up and Go Test (TUG) will be used to measure the gait speed of the participants. This will be performed with a high chair with back and armrests. Participants will begin by sitting down and leaning back in the chair. The participants will rise from the chair by using the arms for support following the command "go". The participants will walk 3 meters in a straight line at their usual speed and then return to the chair and sit down. A stop 
watch will be used to measure the time taken to complete the task. The task duration will be measure up to the moment when the participants will lean back in the chair again. TUG has been shown to have a high inter-observer reliability coefficient $(r=0.99)$ and intra-class correlation coefficient ( $r=0.97)$. ${ }^{18}$

2. Dynamic isometric abdominal test: This test will be used to test the strength of the abdominal muscles and also to determine if the back can be slightly flexed without much pain. The participants will be asked to assume crook lying positions and then tuck in the chin and curl up the trunk and hold that position for $30 \mathrm{sec}$. The test will be graded based on a 5 -criteria scale; 1 ) Normal (score of 5 ), when hands can be placed behind the neck until scapulae clear the table for 20-30sec hold, 2) Good (score of 4), when arms can be crossed over chest until scapulae clear the table for 15-20sec hold, 3) Fair (score of 3), arms straight until scapulae clear table for 10-15sec hold, 4) Poor (score of 2), arm extended towards knees until top of scapulae lift from table for $1-10$ sec hold, 5) Trace (score of 1 ), unable to raise more than the head off the table. ${ }^{25}$

\section{Randomization of Participants}

Eligible participants who provide informed consent will be randomized into one of two treatment groups; Manual Therapy plus Sexual Advice (MT+SA) or Manual Therapy Alone (MTA). A randomization timeline will be prepared by a research assistant who will not have communication with any participant throughout the trial and will be unaware about the study protocol. The randomization series (using block randomization) will be created by the use of SAS 9.4 statistical software (Cary, NC, USA) with the participants likely to be assigned to a group with an equal chance of allocation (see Figure 1, for the study flow chart).

\section{Intervention Procedure}

There will be two intervention groups in this study. The first group which is the manual therapy plus sexual advice (MT+SA) group will receive spinal mobilization and neural mobilization exercise plus sexual advice. The second group which is the manual therapy alone (MTA) group will receive spinal mobilization and neural mobilization exercise without sexual advice. The groups will receive treatments by two independent physiotherapists who are very skillful at manual therapy and are conversant with the techniques. Participants will attend three treatment sessions per week for twelve weeks. The study period will last for 12 months giving by 3 months of data collection and 9 months of follow-up. The full intervention procedure will be described below:

\section{Manual Therapy and Sexual Advice $(\underline{M T+S A})$ Group}

\section{Spinal Mobilisation}

Participants in this group will receive spinal mobilization (MOB) using Mulligan's protocol. ${ }^{26}$ This technique has been investigated to be very effective in the management of individuals with LBP secondary to LDHR. ${ }^{15,27,28}$ This technique will be performed with the participant in a side lying position, with the affected leg uppermost. Participant will be lying facing the treating physiotherapist (PT), and an assistant therapist will be holding the affected leg. The treating therapist will then flex over the participant and place one thumb reinforced over the other on the prominence of the affected vertebra as palpated by taking the posterior superior iliac crest as the reference zone. The therapist will then push down on the chosen spinous process of the affected vertebra and maintain the pressure while the participant will be asked to actively perform Straight Leg Raise (SLR) for the leg held by the assistant therapist if there is no too much discomfort. The position will be maintained for sometime after which the therapist will release the pressure on the spinous process and the participant will be asked to lower his/her supported leg down to the couch. During first visit three (3) repetitions will be applied. On the subsequent visits as the participant improves, the assistant therapist will apply over-pressure on the supported leg of the participant as he/she performs the SLR. This will be also sustained for sometime after which the leg will be lowered to the starting position. Six (6) repetitions will be given on the subsequent visits.

\section{Neural Mobilization Exercise}

Neural mobilization exercise has been shown to be effective as an adjunct therapy in the management of individuals with LBP secondary to LDHR. ${ }^{15,28}$ This exercise will be conducted by carrying out Straight Leg Raise (SLR) to induce tension in the sciatic nerve. The SLR technique will be conducted as per the guidelines of Butler and Jones. ${ }^{29}$ The participant will be in the supine position and comforted with pillows. The therapist will stand on the side to be treated, and raise the affected side perpendicular to the bed with one hand on the popliteal fossa and the other on the patella until sciatic nerve pain is provoked. The limb will then be taken down some few degrees from this painful position. The therapist will start to stretch (mobilize) the sciatic nerve by a series of oscillatory movements toward the ankle dorsiflexion and then reassess progress. To create further tension (i.e., sensitization) into the proximal segment of the affected nerve, hip adduction and internal rotation will be added to the SLR. Many repetitions will be performed depending on the participant's responses and tolerance. As the symptom improves, range of motion will be increased until the maximum range of SLR is obtained. ${ }^{30}$ The position will be held for 30 s and a total of 5 repetitions will be conducted.

\section{Sexual Advice}

1. Planning the Sex: Sex will be planned at a point when patients are in a good mood, and when they have the least Massages will be used to assist in relaxation and mood enhancement.

2. Sexual Environment: Participants will be told to avoid sexual contact on hard surfaces because of the possibility of bruises. Sexual environments should be soft and firm, for example; a firm mattress, cushion chairs, or pillow supports. Sexual contact should be avoided on elevated surfaces to prevent falling when orgasm has been reached.

3. Starting the Sex: Participants will be told to start sex gently and slowly, minimizing rough movements and abrupt changes of positions. Participants will be advised to perform sex by rocking the pelvis and not moving with the Participants will be told that their partners should be the more aggressive ones if 
they do not have low back pain, and participants should also use lumbar supports. A water-soluble lubricant (such as the K-Y Jelly) may be used to reduce painful intercourse. ${ }^{30}$ Participants will be told to avoid prolonged copulation because this may deplete opioid receptors in the brainstem which may cause pain or even predispose to spinal injury. ${ }^{6,7}$

4. Lifting of Partner: Participants will be advised to avoid lifting their partners during sex because lifting is associated with LBP and other musculoskeletal disorders. ${ }^{31}$ The act of stoop lifting is one of the most stressful movements biomechanically and if considered necessary, lifting of a partner during sex is best done when the partner to be carried is placed on a raised platform and has less weight compared to the active partner who should also maintain an upright posture. This precaution will reduce tension generated in the erector spinae muscles, and the force transmitted to the intervertebral discs. ${ }^{31}$

5. Positional Changes: Participants will be examined for directional preference to spinal motions (either flexion-intolerant or extension-intolerant) prior to sexual positions placement. The sexual positions reported by the previous studies ${ }^{12,13}$ will be given to the participants. Based on range of motion, individuals classified as having flexion-intolerance will be given sexual positions that involve back bending. Individuals classified as having extensionintolerance will be given sexual positions that involve forward bending.

\section{Manual Therapy Alone (MTA) Group}

This group will receive spinal mobilization and neural mobilization exercise (similar to previous group) without sexual advice.

\section{Measurement of Adverse Effects}

Adverse (harmful or unpleasant) effects will be measured among the research participants by the investigators. Adverse events will be recorded in standardized manner for each participant at all stages of data collection (4, 8 and 12 weeks). Adverse effects will also be measured using questionnaires during follow-up (6, 9 and 12 months).

\section{Participants' Compliance with Interventions}

The compliance of the participants with their investigators' advice will be assessed at each visit using verbal interviews and recorded in standardized charts. The number of treatment sessions, and the number of missed or cancelled appointments will be also recorded by the investigators. The participants will be instructed not to disclose their individual interventions to themselves and/or to the assessors. Participants will also be informed to suspend all their pain medications to ensure the effectiveness of the techniques and this will however, be monitored periodically. Any trial deviation will be monitored by the investigators and will be discussed appropriately within the research team.

\section{Research Team}

The research team will comprise of six PTs (five orthopedic PTs and one neuro-PT) and three research assistants. The orthopedic PTs (who are trained in manual therapy and are conversant with manipulation) will independently administer the interventions to the experimental groups. One of the research assistants will be responsible for randomization and group allocation while the others will be responsible for outcomes assessment. All research assistants will undergo additional two-day training led by one of the investigators (MSD) where randomization and outcomes assessment will be taught. At each stage of data collection the research team will discuss and review specific issues related to the study.

\section{Integrity of Data}

All data obtained will be scored and entered into a computer spreadsheet by a data analyst who will not be aware of the study design and/or participants randomization/recruitment whatsoever. Data omissions and outliers will be examined to identify entry errors by the data analyst.

\section{Statistical Analysis Procedure}

Data obtained from this study will be analyzed using SPSS 20.0 version software (SPSS Inc., Chicago, Illinois, USA). The demographics and clinical parameters of the participants will be summarized using descriptive statistics. The normality of the data will be assessed using Shapiro-Wilk statistics. Participants' characteristics will be compared at baseline using independent T test. Levene's test will be used to assess homogeneity of variances between groups. Repeated measures ANOVA with intervention (MT+SA and MTA) as the between-subject variable and time (baseline, weeks 4, 8 and 12, months 6, 9 and 12) as the within-subject variable will be used to analyze treatment effects. When significant intra-group differences are detected by ANOVA, Bonferroni post-hoc analysis of variance will be used to assess differences across timelines. The data will be analyzed according to intention-to-treat analysis (ITT) by carrying the last available score forward. Differences between the means will be considered at $5 \%$ probability level $(P<0.05)$ and the value of confidence interval (Cl) will set at $95 \%$.

\section{Discussion}

Many couples experience temporary difficulties with their sexual lives from time to time, regardless of whether one partner has a pain problem or not. When pain enters into the relationship, sexual difficulties may occur because of a combination of factors. Pain, especially low back pain (LBP) may quickly kill sexual arousal because anticipation of the pain can be equally effective in limiting the mood.

Sexual intercourse requires a partner to hold and be held by the other partner, and to exercise joints and muscles. Pain may affect ability to respond sexually and may hinder ability to move freely which may limit the positions that people can get into to have sex. If a patient is frightened of his/her pain increasing 
with movement, then he/she is likely to become tense, and even avoid sexual intercourse. This may strain relationship and may also have serious consequences on the lives of the partners.

Even though, sexual intercourse has been identified as a significant indicator of people's lives and one of the inherent measures of health and disability, it is most often being neglected by healthcare professionals in the rehabilitation of individuals with LBP. This current study will be conducted to determine if the addition of sexual advice in the management of individuals with LBP will yield better outcomes. The major hypothesis of the study was stated as; manual therapy in combination with sexual advice will yield better outcomes than manual therapy alone in the management of individuals with LBP.

The treatments will be administered by two independent physical therapists that are experts in manual therapy techniques and are conversant with the techniques. This decision will be made to minimize investigator bias. The participants will be instructed not to disclose their individual intervention groups to themselves and/or to the assessors. Participants will also be informed to suspend all their pain medications to ensure the effectiveness of the techniques and this will however, be monitored periodically.

This current study will assess both self-report (pain, disability, sciatica bothersomeness and sciatica frequency), and objective outcomes (gait speed and dynamic isometric abdominal strength) to widely track participants' progressions through all stages of data collection, These outcomes will be assessed at baseline, 4, 8 and 12 weeks of intervention. Participants will be discharged home after 12-weeks of intervention and be placed on lumbar stabilization exercises as home regimen. Outcomes will also be assessed at 6, 9 and 12 months of follow-up.

\section{Declarations}

Dissemination of Findings: After completion of the study, findings will be updated in the Pan African Clinical Trial Registry and will be published in a peerreviewed journal.

Author Contribution: M.S.D and A.M.Y developed the idea, M.S.D, S.U.I, U.U.Z and A.M.Y conceptualized the idea, M.F.A and M.S.D sought the fund, M.S.D, A.M.Y, and U.T.S wrote the manuscript.

Research funding: This study has been supported by special grant from the physiotherapy department, Federal Medical Centre, Nguru, Yobe State, Nigeria.

Conflict of interest: The authors do not have any conflict of interest.

Informed consent: Participants will sign informed consent before enrolling in the study.

\section{References}

1. Bahouq H, Allali F, Rkain H, Hajjaj-Hassouni N. Discussing sexual concerns with chronic low back pain patients: Barriers and patients' expectations. Clin Rheumatol. 2013;32(10):1487-1492. doi:10.1007/s10067-013-2299-y

2. Gruenwald I. Sexual Dysfunction in Patients with Chronic Pain. Urol Nephrol Open Access J. 2017;4(6). doi:10.15406/unoaj.2017.04.00147

3. Nikoobakht M, Fraidouni N, Yaghoubidoust M, Burri A, Pakpour AH. Sexual function and associated factors in Iranian patients with chronic low back pain. Spinal Cord. 2014;52(4):307-312. doi:10.1038/sc.2013.151

4. Berg S, Fritzell P, Tropp H. Sex life and sexual function in men and women before and after total disc replacement compared with posterior lumbar fusion. Spine J. 2009;9(12):987-994. doi:10.1016/j.spinee.2009.08.454

5. Nabil G, Muhammad Amin, Yousaf Khan, Sana Ullah JZ. Sexual Activities and Initiation of Body Pain in Men. Am Res J Bio Sci. 2015;1(1):1-2. https://scholar.google.com/scholar?

$\mathrm{hl}=$ en\&as_sdt=0\%2C5\&q=Nabil+G\%2C+Muhammad+Amin\%2C+Yousaf+Khan\%2C+Sana+Ullah\%2C+Jehan+Zeb.+Sexual+Activities+and+Initiation+of+Bo $2 . \& b t n G=$. Accessed January 17, 2020.

6. Garduño-Gutiérrez R, Guadarrama-Bazante L, León-Olea M, Rodríguez-Manzo G. Endogenous opioids mediate the sexual inhibition but not the drug hypersensitivity induced by sexual satiation in male rats. Behav Neurosci. 2013;127(3):458-464. doi:10.1037/a0032332

7. Szechtman H, Hershkowitz M, Simantov R. Sexual behavior decreases pain sensitivity and stimulates endogenous opioids in male rats. Eur $J$ Pharmacol. 1981;70(3):279-285. doi:10.1016/0014-2999(81)90161-8

8. Kinsey AC, Pomeray CE, Martin GP. Sexual Behavior in the Human Female. Vol 10.; 2006. doi:10.1007/s12119-006-1006-4

9. Ross EL, Komisaruk BR, O'Donnell D. Evidence that probing the vaginal cervix is analgesic in rats, using an operant paradigm. J Comp Physiol Psychol. 1979;93(2):330-336. doi:10.1037/h0077555

10. Komisaruk BR, Larsson K. Suppression of a spinal and a cranial nerve reflex by vaginal or rectal probing in rats. Brain Res. 1971;35(1):231-235. doi:10.1016/0006-8993(71)90608-1

11. Whalen RE. Effects of mounting without intromission and intromission without ejaculation on sexual behavior and maze learning. $J$ Comp Physiol Psychol. 1961;54(4):409-415. doi:10.1037/h0046385

12. Sidorkewicz N, McGill SM. Male spine motion during coitus: Implications for the low back pain patient. Spine (Phila Pa 1976). 2014;39(20):1633-1639. doi:10.1097/BRS.0000000000000518

13. Sidorkewicz N, McGill SM. Documenting female spine motion during coitus with a commentary on the implications for the low back pain patient. Eur Spine J. 2015;24(3):513-520. doi:10.1007/s00586-014-3626-y 
14. Faul F, Erdfelder E, Buchner A, Lang A-G. Statistical power analyses using G*Power 3.1: Tests for correlation and regression analyses. Behav Res Methods. 2009;41(4):1149-1160. doi:10.3758/BRM.41.4.1149

15. Das MS, Dowle, P. IR. Effect of spinal mobilization with leg movement as an adjunct to neural mobilization and conventional therapy in patients with lumbar radiculopathy: Randomized controlled trial. J Med Sci Res. 2018;6(1):11-19. doi:10.17727/jmsr.2018/6-3

16. Suresh K, Chandrashekara S. Sample size estimation and power analysis for clinical research studies. J Hum Reprod Sci. 2012;5(1):7. doi:10.4103/09741208.97779

17. Hewitt DJDDJ, McDonald M, Portenoy RKR, Rosenfeld B, Passik S, Breitbart W. Pain syndromes and etiologies in ambulatory AIDS patients. Pain. 1997;70(2-3):117-123. doi:10.1016/S0304-3959(96)03281-2

18. Gautschi OP, Smoll NR, Corniola M V., et al. Validity and reliability of a measurement of objective functional impairment in lumbar degenerative disc disease: The Timed Up and Go (TUG) test. Neurosurgery. 2016;79(2):270-278. doi:10.1227/NEU.0000000000001195

19. Danazumi MS, Ibrahim SU, Abubakar MF, Mohammed A, Zakari UU. Effect of Spinal Manipulation Compared with Spinal Mobilization for Lumbar Disc Herniation with Radiculopathy: Design of a Randomized Clinical Trial with 1 Year Follow-Up. Middle East J Rehabil Heal Stud. 2019. doi:10.5812/mejrh.92770.Methods

20. Weinstein JN, Lurie JD, Tosteson TD, et al. Surgical versus nonoperative treatment for lumbar disc herniation: Four-year results for the spine patient outcomes research trial. Spine (Phila Pa 1976). 2008;33(25):2789-2800. doi:10.1097/BRS.0000000000000088

21. Peul WC, Hout WB van den, Brand R, Thomeer RTWM, Koes BW, Leiden-The Hague Spine Intervention Prognostic Study Group. Prolonged conservative care versus early surgery in patients with sciatica caused by lumbar disc herniation: two year results of a randomised controlled trial. BMJ. 2008;336(7657):1355-1358. doi:10.1136/bmj.a143

22. A.M. B, H.R. SP, M.F. R, J.B. P, R.E. S. Reliability and validity of the visual analogue scale for disability in patients with chronic musculoskeletal pain. Int J Rehabil Res. 2008;31(2):165-169. doi:10.1097/MRR.0b013e3282fc0f93

23. Atlas SJ, Keller RB, Chang Y, Deyo RA, Singer DE. Surgical and Nonsurgical Management of Sciatica Secondary to a Lumbar Disc Herniation. Spine (Phila Pa 1976). 2001;26(10):1179-1187. doi:10.1097/00007632-200105150-00017

24. Grøvle L, Haugen AJ, Keller A, Natvig B, Brox JI, Grotle M. Reliability, validity, and responsiveness of the norwegian versions of the maine-seattle back questionnaire and the sciatica bothersomeness and frequency indices. Spine (Phila Pa 1976). 2008;33(21):2347-2353. doi:10.1097/BRS.0b013e31818047d6

25. Raghav S, Singh A. ROLE OF SWISS BALL EXERCISES IN REDUCING PAIN, DISABILITY AND IMPROVING MUSCLE ENDURANCE IN PATIENTS WITH MECHANICAL LOW BACK ACHE. Orig Res Artic. 2017;5(2):1966-1970. doi:10.16965/ijpr.2017.117

26. Mulligan BR. Spinal mobilisations with leg movement (Further mobilisations with movement). J Man Manip Ther. 1995;3(1):25-27. doi:10.1179/jmt.1995.3.1.25

27. Ahmed N, Khan Z. Comparison of Mulligans Spinal Mobilization with Limb Movement (SMWLM) and Neural Tissue Mobilization for the Treatment of Lumbar Disc Herniation: A Randomized Clinical Trial. J Nov Physiother. 2016;6(4). doi:10.4172/2165-7025.1000304

28. Yadav S, Arora Nijhawan M, Panda P. Effectiveness of spinal mobilization with leg movement (smwlm) in patients with lumbar radiculopathy (I5 / s1 nerve root) in lumbar disc herniation. Int J Physiother Res. 2014;2(5):712-730. www.ijmhr.org/ijpr.html. Accessed March 3, 2020.

29. Butler DS. David S. Butler - Mobilisation of the Nervous System. (Jones MA, ed.). Churchill Livingstone; 1991.

30. Hall T, Beyerlein C, Hansson U, Lim HT, Odermark M, Sainsbury D. Mulligan traction straight leg raise: A pilot study to investigate effects on range of motion in patients with low back pain. J Man Manip Ther. 2006;14(2):95-100. doi:10.1179/106698106790820782

31. Cole MH, Grimshaw PN. Low back pain and lifting: A review of epidemiology and aetiology. Work. 2003;21(2):173-184.

\section{Figures}




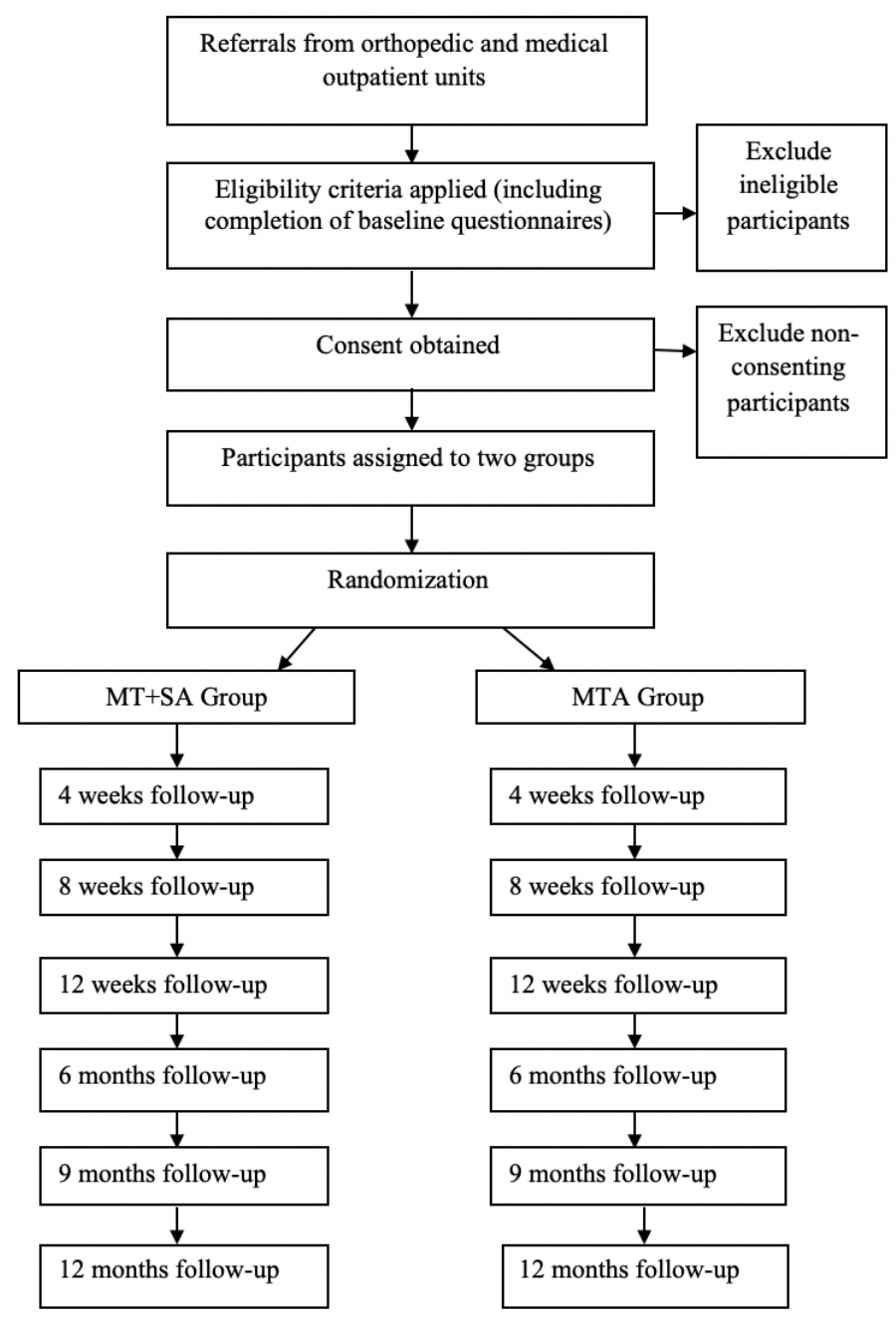

Figure 1

Study Flow Chart 